\title{
Enabling WiMAX Video Streaming
}

\author{
M. Fleury, R. Razavi, S. Saleh, L. Al-Jobouri and M. Ghanbari \\ University of Essex \\ United Kingdom
}

\section{Introduction}

IEEE 802.16 (WiMAX - Worldwide Interoperability for Microwave Access) (Andrews, 2007; Nuaymi, 2007) is a broadband wireless technology that allows rapid deployment of video streaming services, including Video-on-Demand (VoD), multicast varieties of IPTV (TV over the Internet Protocol) and broadcast, digital TV (Meloni. 2008). Streaming assumes limited buffering of data for video-rate display, rather than video file download, video messaging services, or hybrids for video clip download in which display is overlapped with download of video chunks. In these services, delay is important but not as important as in interactive, conversational services such as video-phoning and video conferencing (Wenger, 2006), in which one-way delay should ideally approach (but not reach in practice) $100 \mathrm{~ms}$. To illustrate the issues, the Chapter considers the possibility of a simple but effective adaptive modulation scheme for WiMAX offering unequal error protection (UEP) of video according to frame type (Ghanbari, 2003). To allow channel swapping when broadcasting TV channels in a multiplexed stream and to emulate VCR functionality in VoD, spatially-coded (Intraframe) I-frames are often inserted around every half-second of video. I-frames also act to anchor the decoder in case of loss of synchronisation and consequent propagation of distortion. Other frame types include Predicted (P-) frames, which are backwardsly predicted from I- or other P-frames, and Bi-predicted (B-) frames, which as the name suggests can be predicted from I- and P-frames in either temporal direction. B-frames are not employed in the Baseline profile of the state-of-the-art H.264 codec (Richardson, 2003) in order to reduce computation levels on mobile devices but can be employed in streaming,when latency is less of an issue.

In the UEP scheme, by monitoring the input video bitrate, a burst profile is selected that offers a lower rate than the arrival rate to more important anchor frames. If I-frame data is protected then the less important $\mathrm{P}$-and B-frame data rates must compensate, which occurs in the scheme through selection of one or more WiMAX burst profiles offering higher data rates. Allocation depends on the congestion level indicated by transmit buffer fullness. For example, low buffer fullness may allow some P-frame data to be protected while high buffer fullness requires some P-frame data to be allocated to a still higher data rate.

An important consideration for WiMAX is the effect of propagation conditions in the outdoor environment. The Stanford University Interim (SUI) channel model adopted in the IEEE 802.16 standardisation is an extensive and complete channel model, implemented by the authors within the well-known ns-2 simulator, and used along with the NIST WiMAX 
simulation module. The behavior of the WiMAX modulation modes along with physical layer characteristics under the SUI model will be discussed herein. An effective UEP scheme is one that combines simplicity of implementation with compelling gains in video quality. One such algorithm is proposed in the Chapter. Applying an additive-increase and multiplicative-decrease algorithm to the choice of burst profile, results in many decibels gain in delivered video quality and significant gains in delay/jitter reduction over a nonadaptive modulation scheme.

Considerable research has taken place into bandwidth allocation, packet scheduling and queuing management for WiMAX service classes. The Chapter evaluates Active Queuing Management techniques for uplink scheduling, as this topic has received comparatively limited attention in the research community. In the WiMAX standard, queuing discipline and queue scheduling are left open to the developer for reasons of commercial differentiation. Unlike work conserving queuing mechanisms, AQM drops packets in anticipation of growing congestion. The Chapter will examine the most effective strategy for WiMAX uplink management.

The current H.264 codec outputs compressed data encapsulated as Network Adaptation Layer Units (NALUs) which are mapped onto MAC Packet Data Units (MPDU), before transmission over the WiMAX link. However, there are different ways to aggregate or segment the H.264 NALUs according to the MPDU size, which may be fixed or variable. As an alternative, UEP can also be based upon H.264 data partitioning or differential error concealment can be applied. In data partitioning, the more important parts of the compressed bitstream are collected in separate NALUs, so that in H.264 up to three different types of NALU can be generated and the more important partition protected with increased application layer Forward Error Correction (FEC). Our research is at an exploratory stage for this topic, and Section 5 in conjunction with Section 2 introduces the topic. Section 3 reports research on the UEP scheme, whereas Section 4 reports investigations into WiMAX queue management with video transport in mind. Section 6 concludes this Chapter.

\section{WiMAX background}

This Section briefly introduces WiMAX in preparation for descriptions of recent developments in video streaming in later Sections. It is assumed that general descriptions of WiMAX and its applications will be available to the reader from other sources, some of which appear in the references to this Chapter (Andrews et al. 2007, Nuaymi, 2007). For coverage of standard video codecs in general, we can but recommend one of the contributors to this Chapter's textbook (Ghanbari, 2003), while for H.264 in particular (Richardson, 2003) is helpful. For video transport over wireless (Sadka, 2002; Schaar \& Chou, 2007) are recommended.

WiMAX is a competitive solution for 'last-mile' broadband access compared to cable, digital subscriber line (DSL) or fiber, because of the low cost of deploying WiMAX over a wide area. As such WiMAX may be suitable for depolyment in rural areas (Hillestad et al., 2006) in which there is limited previous communication infrastructure, particularly within the developing world. Moreover, rural areas do not present the same problems of wireless propagation as exist in urban areas. There are two main standards for WiMAX: the first one is IEEE 802.16-2004 for fixed wireless connections and the second one is IEEE 802.16-2005 for mobile wireless connections (Yan \& Kavehard, 2006). WiMAX operates within two licensed 
spectrum ranges: 1) from 2.5 to $2.7 \mathrm{GHz}$ in the USA and 2) a range from 3.4 to $3.7 \mathrm{GHz}$ in Europe and the rest of the world. Fixed WiMAX (IEEE 802.16-2004) is suitable for the frequency band $2-11 \mathrm{GHz}$ and the theoretical range is up to 30 miles for line-of-sight (LOS) communication with data rates up to $75 \mathrm{Mbps}$ and for non-line-of-sight (NLOS) the theoretical range is 5 miles with data rates up to $5 \mathrm{Mbps}$. WiMAX supports different duplex modes (Tariq et al., 2007): 1) time division duplex (TDD), and 2) frequency division duplex (FDD) and 3) half-frequency division duplex (H-FDD). For TDD, the system employs the same frequency to transmit over the downlink (DL) and uplink (UL) but within different time slots. Projections normally assume (Yun \& Kavehard 2006) that the DL will have the predominance of the time slots (see Section 2.1 for sub-frame structure), though the allocation could vary dynamically. In FDD, the system requires two different frequencies, while for H-FDD the system uses two different frequencies with different time slots. FDD is most suitable for symmetric loads such as voice channels, while TDD is more suitable for asymmetric loads such as video streaming.

WiMAX has two main components, Base Stations (BSs) and Subscriber Stations (SSs), BSs uses downlink to transmit while the SSs use the UL to transmit. The 802.16 standard can work in a point-to-point (PTP), point-to-multipoint (PMP), and mesh topology modes. By using omni-directional or directional antenna (Andrews et al., 2007) the effective range of the system can be increased.

\subsection{WiMAX frame structure}

The key to understanding how video streams may map onto the WiMAX frame structure is knowledge of the WiMAX frame structure. However, those readers familiar with this background material may wish to proceed to Section 3.

The structure takes different forms across the protocol stack layers. The physical layer of WiMAX is based on the physical layer of Orthogonal Frequency Division Multiplexing (OFDM), which gives WiMAX immunity to frequency selective fading and intersymbol interference. Assuming the more spectrally efficient TDD mode, for the fixed standard (IEEE 802.16-2004) the size of FFT is limited to 256 subcarriers, 192 of the being subcarriers used for data carrying, with 8 subcarriers used as pilot subcarriers for synchronization and channel estimation, while the rest of the subcarriers are used as guard subcarriers.

Figure 1 shows the TDD frame of fixed WiMAX (Andrews, 2007). This frame is divided into a DL sub-frame and UL sub-frame. The frames are separated by a receive-transmit transition gap (RTG) and the sub-frames are separated by transmit-receive transition gap. The DL sub-frame starts with a preamble and then a Frame Control Header (FCH). The FCH contains the DL- Mobile Application Part (MAP), UL- MAP, downlink channel descriptor (DCD), uplink channel descriptor (UCD) and one or more DL-bursts. The other DL-bursts are used for data .The UL sub-frame starts with ranging slots, Contention for Bandwidth Request (CBR) and UL-bursts.

One of the attractive features of the Medium Access Control (MAC) layer of WiMAX that it is able to construct and transmit fixed and variable-sized frames. The MAC layer has three sublayers; these sublayers interact with each other via service access point (SAPs). The service-specific convergence sublayer achieves the mapping or transformation of external network data by the help of the SAP. The common part of the SAP receives information from the MAC Service data units (MSDUs), which are packed into the payload field to form MAC protocol data units (MPDUs). 


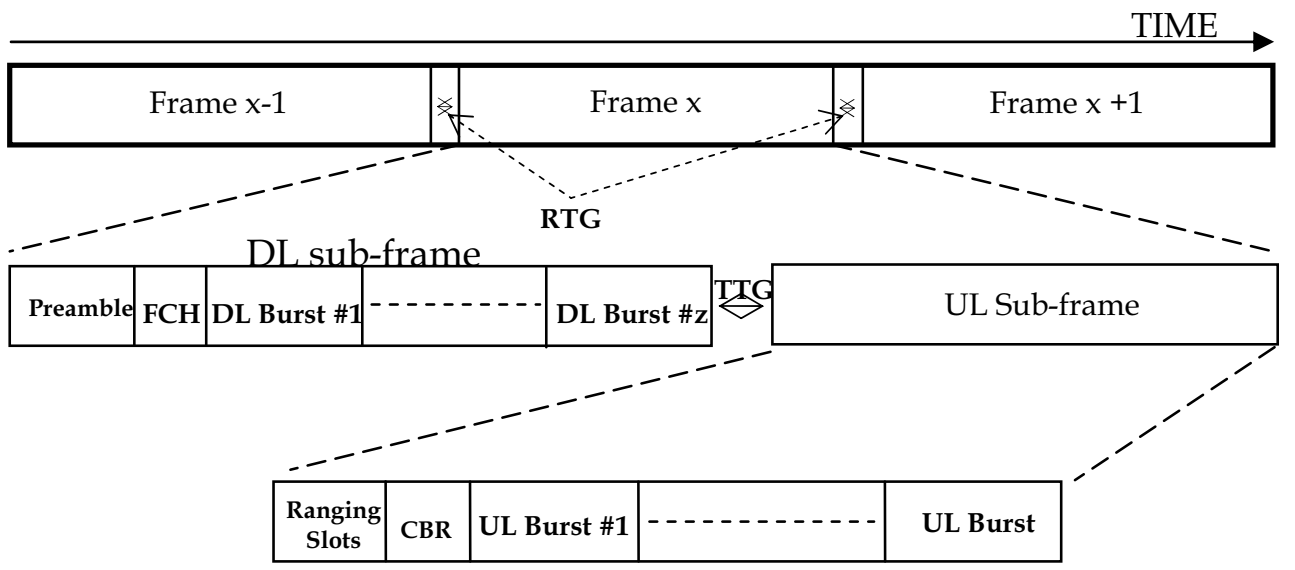

Fig. 1. Fixed WiMAX OFDM-TDD frame stucture

The common part sub-layer is able to pack MSDU into MPDU in different ways (more than one complete or partial). This ability is called aggregation. Figure 2 shows the ability of the MPDU to accommodate more than two MSDUs but not three. As a result, the remaining part of a third MSDU is packed with the previous two MSDUs to fill the remaining payload field to prevent wastage of resources. Feedback from an SS and on-air timing slots decide the payload size. Aggregation in the payload of MPDU is indicated by 'type' bit in the generic MAC frame header (GMH). Subheaders (Ahson \& Ilyas, 2008) consist of two bits of fragment control (FC), three bits of fragment sequence number (FSN) when the MSDU is fragmented and three bits of length field. To indicate the start of the next subheader in the payload. FC is set to 00 when the MSDU is not fragmented and if the MSDU is fragmented, FC is set to 10,01 or 11.

The common part sublayer also has the ability (Ahson \& Ilyas, 2008) to fragment an MSDU across multiple MPDUs. Figure 3 shows how a portion of a single MSDU taking up the entire payload of an MPDU. When the size of the transmitted packet of MAC payload is too small to accommodate a complete MSDU, the MSDU is fragemented and a fragment is placed in another MPDU. Here, the FC bit is set to 01 if it is the last fragment of the MSDU; 10 if it is the first fragment of the MSDU; and if it is the MSDU is fragmented, FC is set to 10, 01 or 11 . 


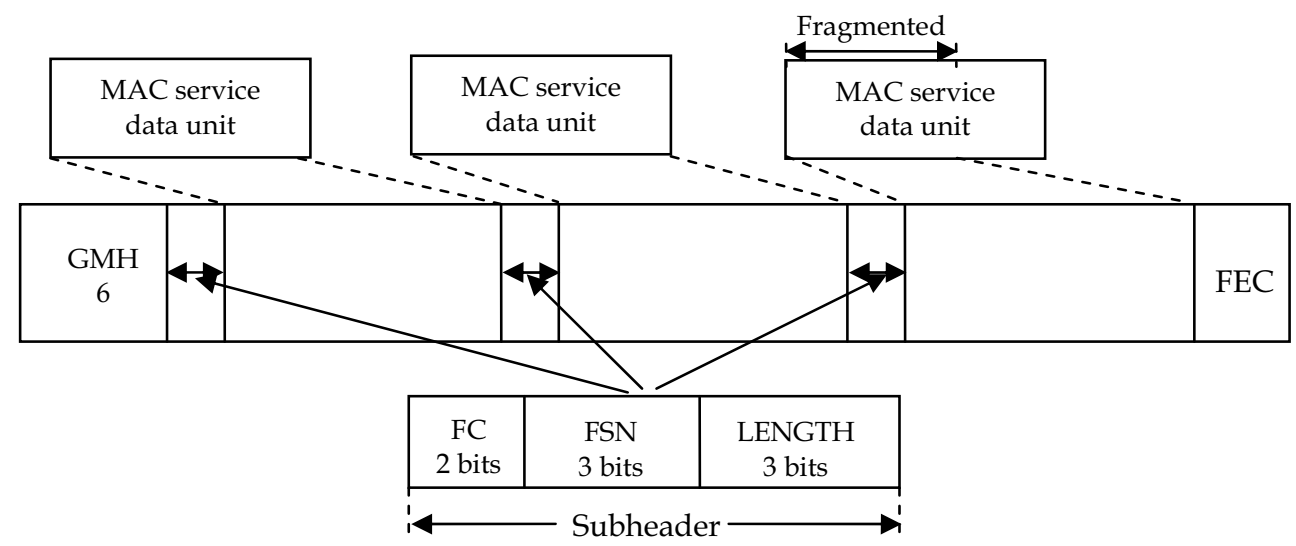

Fig. 2. Multiple MSDUs forming one MPDU

\section{Unequal Error Protection}

\subsection{WiMAX modulation}

Fixed WiMAX (IEEE 802.16d) employs OFDM to counter the effect of inter-symbol interference (ISS) when channel delay spread, $\tau$, is large relative to symbol time $T_{s}$, due to high data rates and multipath fading. OFDM achieves this by dividing the input into $n$ subcarriers such that that $n T_{s} \gg \tau$. To avoid any ISI through sub-carrier roll-off a cyclic prefix $(\mathrm{CP})$ acts as a guard interval between bursts greater than $\tau$. In fact, addition of a CP allows the application of an Inverse Fast Fourier Transform (IFFT) to act upon the data stream as if performing a cyclic convolution to form the $n$ sub-carriers.

As described in Section 2, through OFDM, IEEE 802.16d NLOS supports 256 sub-carriers in a TDMA/TDD system, with scheduling of the sub-carriers at a BS, with notification of scheduling to SSs in the frame control header. Appropriate symbol mapping to the complex frequency domain allows selection of the modulation mode prior to IFFT. Within a DL subframe, data bursts are ordered according to the robustness of the burst profile. Therefore, depending on the adaptive modulation mode chosen, data can be mapped onto one or more DL-bursts, as detailed in Section 2.1. Table 1 shows the 7 burst profiles, with change of Forward Error Coding (FEC) rate achieved by puncturing upon an inner non-recursive convolution code (CC) (with symbol-level protection from a fixed outer Reed-Solomon code). 


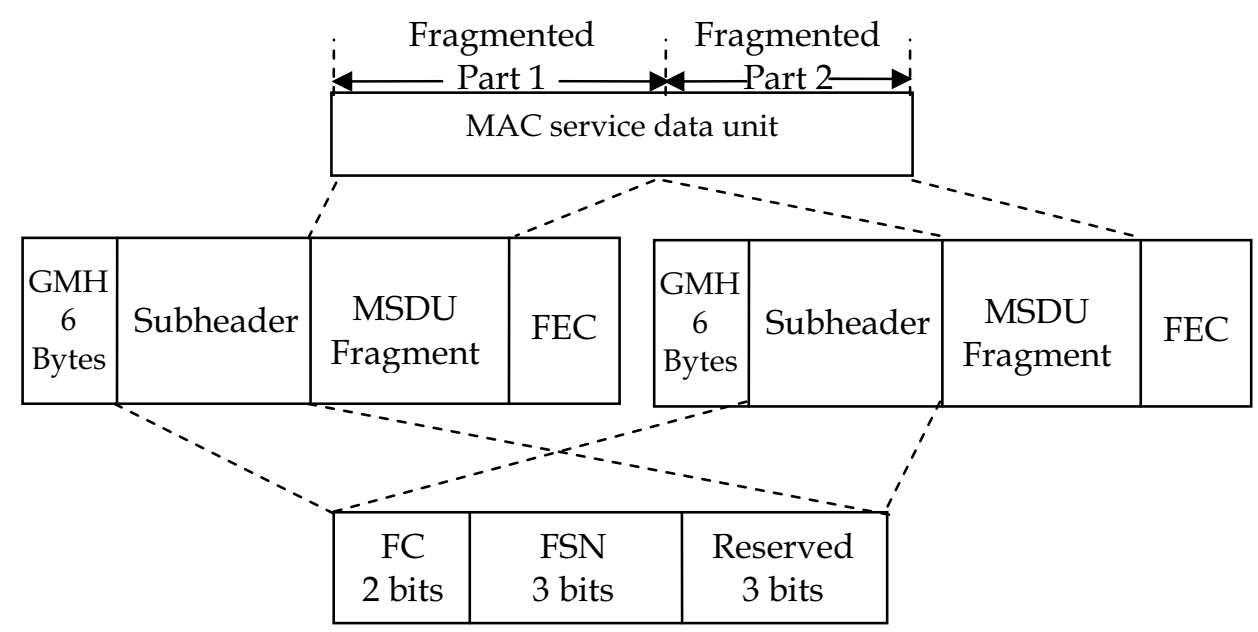

Fig. 3. Single MSDU fragmented across more than one MPDU

Selection of the appropriate WiMAX modulation mode in practice very much depends on channel estimation. WiMAX provides sub-carrier pilot tones for this purpose, as well as optional data burst preamables and/or midambles. Various schemes exist in WiMAX for placement of pilot tones, but other than noting this is important for channel estimation (Negi \& Cioffi, 1998), the topic is beyond the scope of this Chapter. It is also possible (Juan et al., 2007) to monitor the downlink channel at the receiver and return the results in the UL sub-frame.

\begin{tabular}{|l|l|l|}
\hline Profile & Modulation mode & Coding rate \\
\hline 1 & BPSK & $1 / 2$ \\
2 & QPSK & $1 / 2$ \\
3 & & $3 / 4$ \\
4 & 16QAM & $1 / 2$ \\
5 & & $3 / 4$ \\
6 & 64QAM & $2 / 3$ \\
7 & & $3 / 4$ \\
\hline
\end{tabular}

Table 1. Modulation profiles under fixed WiMAX, BPSK = Binary Phase Shift Keying, QPSK = Quadrature PSK, 16QAM = 16 Quadrature Amplitude Modulation

\subsection{WiMAX propagation model}

Broadband wireless propagation in general is introduced in (Anderson, 2003). There are a number of empirical models such as IEEE 802.16 (SUI), COST-231 Hata model, MMDS Band Empirical Path Loss. The channel model set selected by us were the IEEE 802.16 Stanford University Interim (SUI) models (Ercge et al., 2001; Hoymann, 2005). From these, channel two out of six empirical channels designed to cover terrain in the US. SUI-2 models a flat 
terrain with light tree density and low Doppler spread. The cell radius is assumed to be 6.4 $\mathrm{km}$. Antenna height at the BS is $15.24 \mathrm{~m}$, beamwidth $120^{\circ}$ and at the SS is $3.048 \mathrm{~m}$, beamwidth $50^{\circ}$, with vertical polarization only. The SUI models provide generic system development modelling, as does COST-231. Studies such as (Milanovic, 2007) have concluded that the SUI model provides good accuracy for NLOS propagation but should not be relied upon for LOS propagation, when other models may be preferable.

Table 2 summarizes settings in the Standard for a $7 \mathrm{MHz}$ licensed bandwidth, for which we chose to simulate the $5 \mathrm{~ms}$ frame duration. Notice that profile 1 is reserved for burst mapping data. The SSTTG/SSRTG are Subscriber Station Transmit (Receive) Transition Gaps. The achievable throughputs, with hard CC decoding, according to burst profile are shown in Figure 4. For SUI-2, a WiMAX CP of 1/32 is most appropriate, yielding a relatively limited delay spread of $0.2 \mu$ s. Figure 5 shows the bit error rate (BER) response of the differing burst profiles for SUI-2. For a given burst profile, there is a rapid fall-off in acceptable BER, once outside a narrow Signal-to-Noise (SNR) range. There are also uneven jumps in SNR and, thus, selecting a burst profile to achieve a target BER is difficult.

\begin{tabular}{|l|l|}
\hline Parameter & Value(s) \\
\hline OFDM symbol duration & $34 \mu \mathrm{s}$ \\
Channel bandwidth & $7.0 \mathrm{MHz}$ \\
Frame duration & $5 \mathrm{~ms}, 10 \mathrm{~ms}$, or $20 \mathrm{~ms}$ \\
Maps modulation & BPSK \\
FEC code & RS-CC \\
SSTTG/SSRTG duration & 1 OFDM symbol \\
\hline
\end{tabular}

Table 2. Standardized system parameters

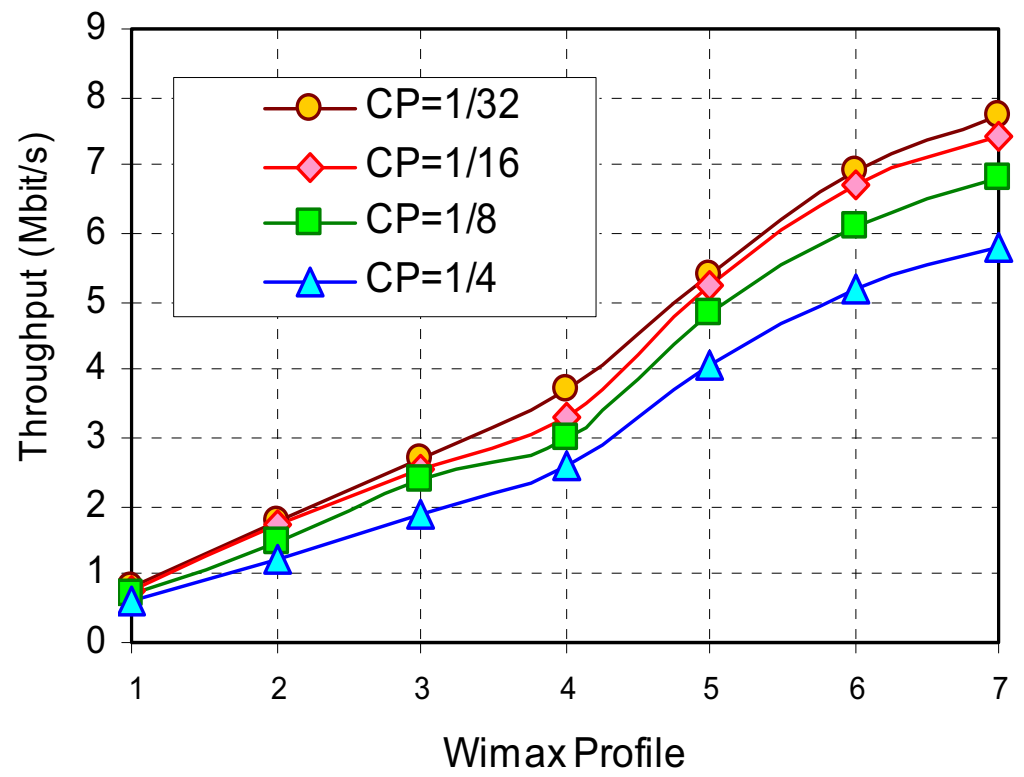

Fig. 4. Throughput for various $\mathrm{CP}$, with $7 \mathrm{MHz}$ bandwidth and 7:3 ratio of DL:UL 


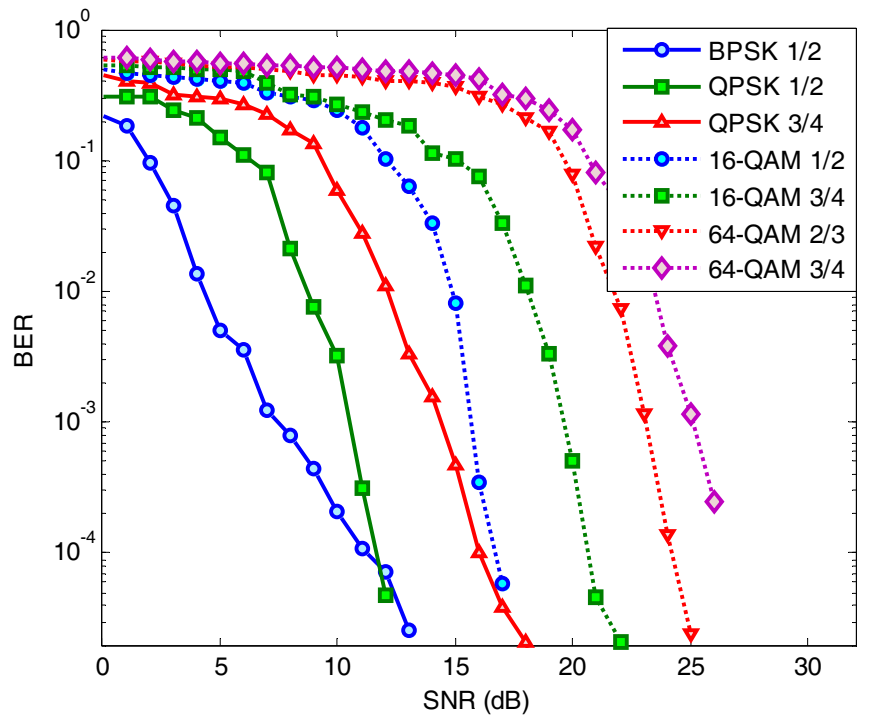

Fig. 5. BER over an SUI-2 channel for $\mathrm{CP}=1 / 32$ with $7 \mathrm{MHz}$ bandwidth and various burst profiles.

\subsection{Adaptive modulation}

Adaptive modulation schemes for video UEP purposes have been considered in general (Wenger, 2003) or for particular wireless technologies (Razavi, 2008). Frequently, video UEP is combined with cross-layer techniques to vary error protection, for example in (Chen et al., 2001)). However, WiMAX already supports variable error protection through changes in the coding rate at the data-link layer. Examples of video UEP for WiMAX are apparently few. In (Juan et al., 2007) for a scalable video stream, the stream was partitioned between different connections to the same station, using the mobile version of WiMAX, IEEE 802.16e. This method did employ different burst profiles within the same UL sub-frame. However, the wireless channel model was the well-known Gilbert-Elliott, two-state Markovian model, which though suitable for modelling 'bursty' errors in indoor environments, fails to take into account the impact of terrain, precipitation, or attenuation in outdoor environments. For data transport in general, adaptive modulation is an important technique with recent attention (Hanzo \& Choi, 2007) concentrated on adaptive OFDM to support constant error rates.

\subsection{Unequal error protection with adaptive modulation}

The general adaptive modulation procedure that we employ, which is repeated over time, is summarized in Figure 6. Arriving compressed video data is monitored to form a timeaveraged estimate of the arrival rate. By means of this estimate the lowest modulation profile that can satisfy the rate at that time is determined. The protection policy across the video packet frame types is updated according to send buffer fullness at the WiMAX BS. All I-frame packets are protected with a robust profile but, to compensate, other packets are accorded less protection according to a policy, which evolves with buffer fullness. 
The first step in our UEP algorithm is to apply a moving average filter to the arriving video data in order to form an estimate of the required WiMAX burst profile, $M$, that fulfils the input data rate. The input video rate is monitored through a linear predictive filter (LPF) (Honig \& Messerschmitt, 1990) over 50 Group of Pictures ${ }^{1}$ (GOPs). For example from Figure 1, for an average $2.5 \mathrm{Mbps}$ input rate, $M$ would be set to profile three, as this most closely meets the data rate requirements. Notice that in this instance it is possible to choose the profile without additional channel estimation, avoiding inaccuracies in estimation.

Upon determination of $M$, the profile $M-1$ is invariably selected for all arriving IP packets bearing I-frame data. Relative allocation of P- and B-frame packets to the different burst profiles is now decided by the normalized buffer fullness, $B$. The fullness is updated after an interval, empirically determined to avoid excessive fluctuations in burst profile selection policy. Assume an available capacity index (ACI) initially set at 1 within a range $[0,1]$, where 1 signifies an empty buffer. Changes to the ACI are only made if buffer fullness either exceeds a high threshold or drops below a low threshold. If $B$ falls below the low threshold $\mathrm{ACI}$ is additively increased by $\mathrm{a}$, which has the effect of increasing the number of packets sent at a lower data rate. If $B$ rises above the high threshold then $\mathrm{ACI}$ is multiplicatively reduced by factor $\beta$ which correspondingly backs off the sending rate to avoid packet loss. This is achieved by favoring higher data rates through higher burst profiles.

Transmit buffer fullness is a general indicator of congestion from input traffic arriving at the BS and of channel conditions. As such the algorithm can be applied across the burst profiles and according to traffic sharing the TDMA frame.

\subsection{Experimental verification of UEP adaptive modulation}

The well-known ns-2 network simulator augmented with the NIST WiMAX module was employed to model the system. The simulations were repeated and averaged over fifty runs to ensure convergence in the face of a high channel error rate. WiMAX's frame duration was set to $5 \mathrm{~ms}$. The video-bearing DL sub-frame was allocated $70 \%$ of the frame duration. In order to isolate the impact of the video stream upon buffer fullness, the arriving video stream was the sole input into the BS send buffer. Thus, the DL sub-frame was occupied by a set of bursts according to the allocation by frame type packet in the UEP algorithm. Data is always assumed to be available to fill the remaining duration of the DL sub-frame as well as the UL sub-frame, though as mentioned, it does not occupy the send buffer. Table 3 reports other settings for the UEP algorithm during simulations. Buffer size was set to 50 packets as also used in (Li \& Schaar, 2004) for video streaming over wireless.

1 In this Chapter, the terms 'picture' and 'frame' are employed interchangeably as we assume progressively scanned video throughout. 


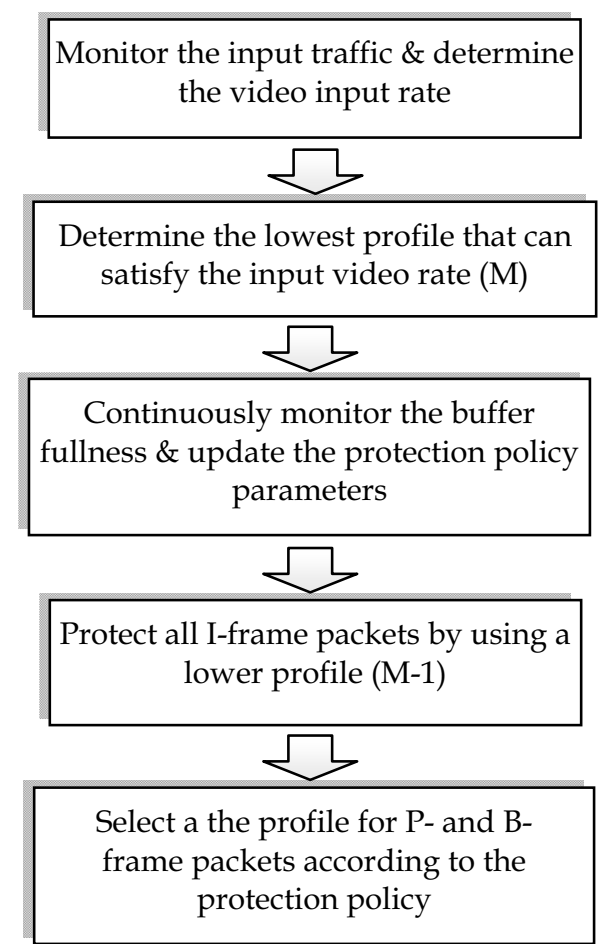

Fig. 6. Overview of the UEP scheme

\begin{tabular}{|l|l|}
\hline Parameter & Setting \\
\hline Buffer capacity & 50 IP packets \\
Update interval & Every 50 IP packets \\
Low threshold & 0.20 \\
High threshold & 0.80 \\
a, $\beta$ & $0.05,1.25$ \\
\hline
\end{tabular}

Table 3. Parameter settings for the UEP algorithm.

To establish the efficiency of the algorithm, a non-adaptive scheme was compared with the UEP scheme. The LPF was again used to select the appropriate profile from Table 1. However, unlike in the UEP algorithm, the same profile was selected for all arriving videobearing IP packets.

Three MPEG-2 video clips appear in the experiments. All were encoded at a variable bit-rate (VBR) with a target rate of $2.5 \mathrm{Mbps}$ for $40 \mathrm{~s}$. Clip 1, 'News', contains some motion, showing a newsreader and changing backdrop. Clip 2, 'Friends', an excerpt from the well-known situational comedy, is more complex, with scene cuts and moderate motion. Finally, clip 3 , 'Football', contains considerable motion and is of higher complexity. Most legacy video is encoded in MPEG-2 and it remains widely used in the broadcasting industry. Peak Signal- 
to-Noise Ration (PSNR) ${ }^{2}$ was found by reconstructing with a reference MPEG-2 decoder. The display rate was $25 \mathrm{~Hz}$, resulting in 1000 frames in each simulation run (averaged over fifteen runs). The source video format was European Source Input Format (SIF) $352 \times 288$ pixel/frame, with a GOP structure of $\mathrm{N}=12$, and $\mathrm{M}=3$, i.e. an IBBPBBP...structure. Error concealment was by previous frame replacement, which is a simple but standard scheme. The scenario modelled assumed arriving video-bearing IP packets at the BS containing, for error resilience purposes, one slice per packet. A slice in MPEG-2 takes-up a row of macroblocks.

Figure 7 shows packet loss rates for the 'News' clip under the SUI-2 channel model at several different average SNR settings ${ }^{3}$. Due to the adaptive modulation scheme, not only does the total packet loss rate considerably reduce but the proportion of lost B-frame packets increases, resulting in preservation of the vital I-frame packets. The loss rate of I- frames is always less than $1 \%$ with the adaptive scheme. As an example, at $17 \mathrm{~dB}$ SNR, the loss rate of B- and P-frames combined is $24.68 \%$ without adaptation and $15.76 \%$ with. Because the adaptive scheme allocates I-frame packets to a more robust profile, the result is a general reduction in the number of lost packets. The presumably increased loss rate of packets allocated to a less robust profile does not match the gain from allocation to the more robust profile.

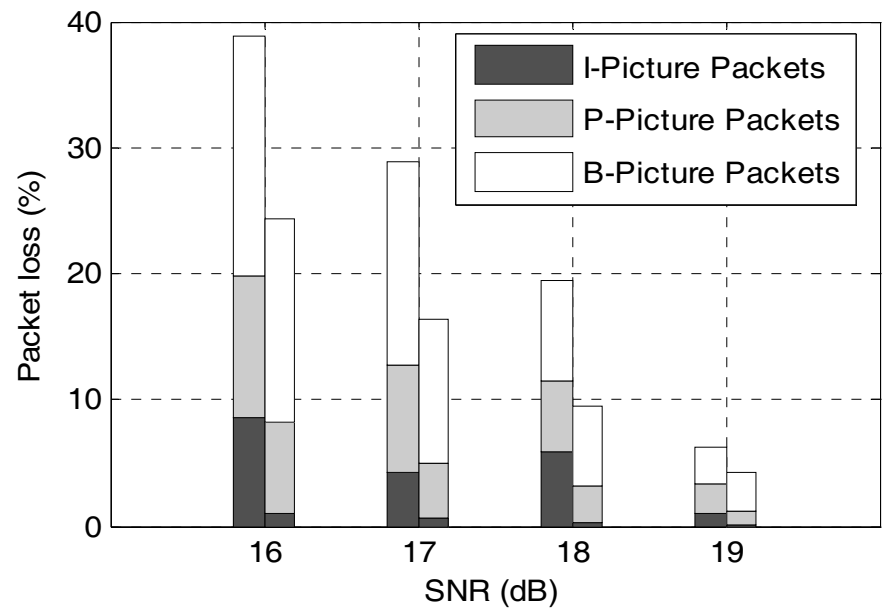

Fig. 7. Comparison of IP packet loss rates for (lefthand) non-adaptive modulation; and (righthand) adaptive modulation for the 'News' clip under the SUI-2 channel model.

Figure 8 compares the resulting video quality (luminance PSNR) between non-adaptive and adaptive modulation with UEP. The effect of UEP is to accentuate the gain from the reduction in packet losses from adaptive modulation. At $16 \mathrm{~dB}$, with non-adaptation all clips would be unwatchable, while the UEP scheme quality verges on the acceptable by virtue of an almost $10 \mathrm{~dB}$ improvement. At SNR of $17 \mathrm{~dB}$, the video quality is good when UEP is

\footnotetext{
2 PSNR = $10 \log \left(\mathrm{MAX}^{2} / \mathrm{MSE}\right)$, where MAX is the maximum intensity value possible for a pixel, and $\mathrm{ME}$ is the pixel-wise mean square error between a reference frame and the frame under test.

${ }^{3}$ Lower SNRs result under this model result in unacceptable video quality and are not presented.
} 
applied but is poor otherwise. When SNR improves, as might be expected the relative advantage of UEP declines. However, UEP at $19 \mathrm{~dB}$ SNR still contributes to several $\mathrm{dBs}$ improvement in video quality, which is now excellent at $40 \mathrm{~dB}$.

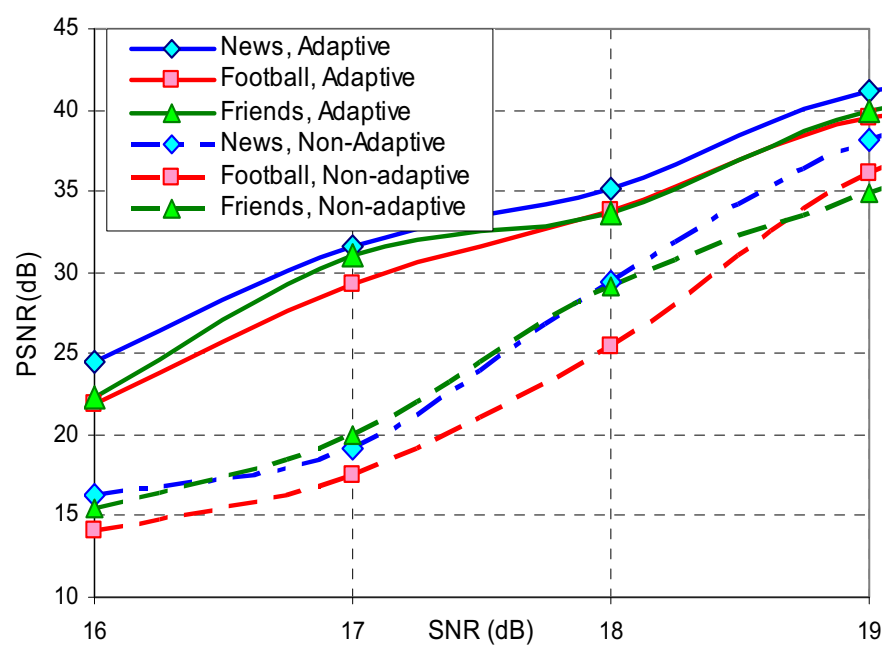

Fig. 8. Comparison of video quality for three video clips of increasing complexity using adaptive and non-adaptive modulation under the SUI-2 channel model

Figure 9 shows that delivered video quality is somewhat sensitive to selection of $\alpha$ and $\beta$ in the adaptive algorithm. The Figure shows variation of the chosen parameters with a drop in PSNR if these parameters are not chosen well. Because the additional plots in Figure 7 occur with larger values for $\alpha$ and $\beta$ then the changes in rate would tend to be more dramatic, with more of a risk from packet loss or under- utilisation of the available bandwidth. However, no dramatic variation in quality occurs, indicating the robustness of the algorithm.

The impact of the adaptation technique on the delay and delay jitter experienced by the application is now considered. If not probably addressed, the delay and delay jitter (variance of delay) could degrade the performance of an application. Click-on-Web-clip users are especially delay intolerant, as are interactive applications, when the delay should be no more than $100 \mathrm{~ms}$ in total (Wenger, 2003). Longer start-up delay requires larger playout buffers and possible overflow if not provided or underflow if excessive jitter occurs. Unfortunately, adaptive buffers sensitive to changes in network traffic can result in disconcerting playout discontinuities. By utilizing WiMAX's ARQ provision, IP packets contained in a burst received in error after application of WiMAX FEC are retransmitted in subsequent DL sub-frames up to a pre-determined retransmission limit (RL), after which the data is discarded if not successfully received. 


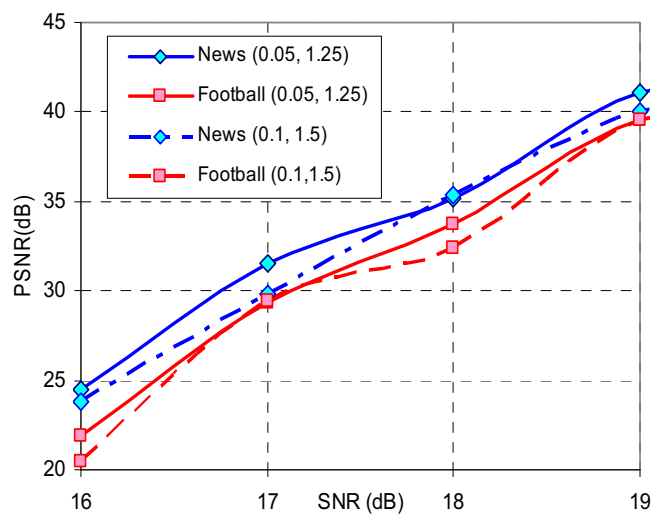

Fig. 9. Comparison of video quality for two video clips of increasing complexity using adaptive modulation under the SUI-2 channel model with variation of $\alpha$ and $\beta$ parameters.

In Figure 10 (for the 'News' clip) the RL is varied and a comparison is made of the resulting delay, with and without the presence of UEP. In all cases, delay is significantly greater without adaptive modulation than it is with. Particularly for $R L=1$, average SNR, though the delay of all schemes significantly improves for SNRs over $18 \mathrm{~dB}$. From Figure 7, it was observed that the packet loss figures were generally reduced for the adaptive modulation scheme, irrespective of the impact of UEP. Though throughput is reduced for those packets transmitted under a robust profile, this delay is more than compensated by the reduction in packet loss, which also reduces the need to retransmit.

As will be noticed from Figure 11, the levels of delay are quite significant for all schemes at lower SNR, especially when it is considered that there will be additional delay across the core network prior to final hop WiMAX delivery, as well as any delay at the codec for realtime applications. Again adaptive modulation always alleviates jitter, Figure 11, and this is as well because levels of jitter are relatively high.

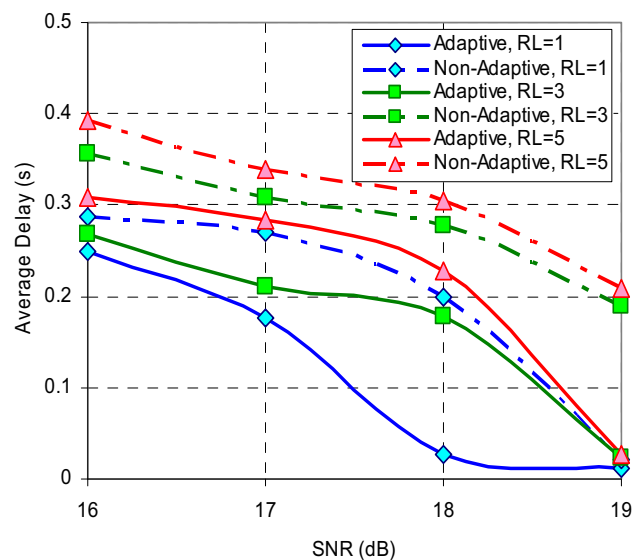

Fig. 10. Comparison of IP packet delay for adaptive and non-adaptive schemes across a WiMAX link 


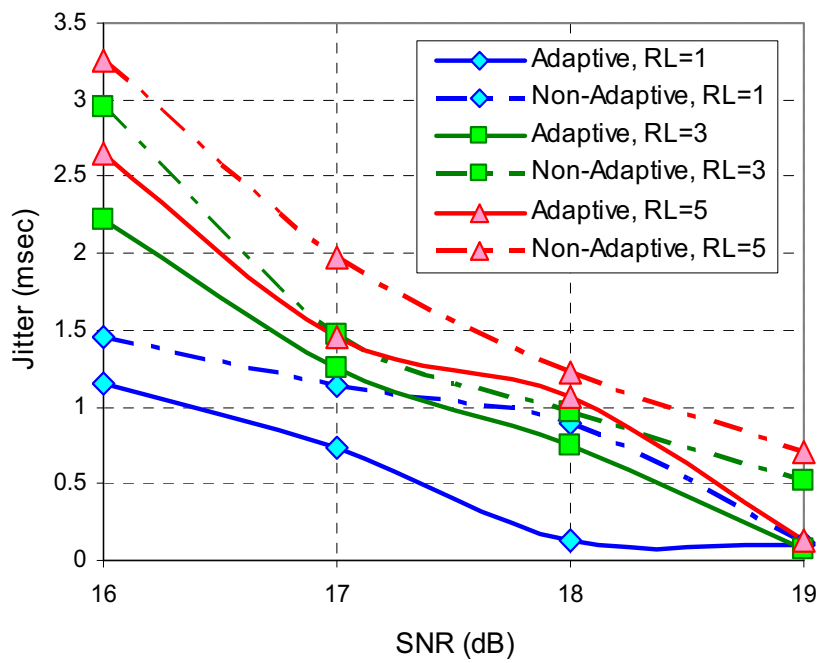

Fig. 11. Comparison of IP packet jitter for adaptive and non-adaptive schemes across a WiMAX link

\section{Queue management for video streaming}

\subsection{WiMAX uplink scheduling and management}

WiMAX in all its versions (IEEE 802.16 parts a-e) does not specify queue management algorithms at the BS or SS, allowing these, along with bandwidth allocation at the BS and packet scheduling at the SS, to be designed by vendors as a means of commercial differentiation. Choice of queuing discipline is sensitive to application type and service class whether it is implemented on the BS or the SS. WiMAX networks support multiple service classes to accommodate heterogeneous traffic with varying requirements. In particular, these are Unsolicited Grant Service (UGS), extended real-time Polling Service (ertPS), realtime Polling Service (rtPS), non-real-time Polling Service (nrtPS), and Best effort (BE). UGS is designed for synchronous services such as legacy voice without silence suppression. The ertPS class (Zhang et al., 2006) is intended for voice with silence suppression, when a SS may not have data available upon polling by the BS. The rtPS class is most suitable for real-time video services, particularly for Variable Bitrate Video (VBR), which is employed to maintain delivered video quality but may lead to 'bursty' arrival rates.

Figure 12 shows how an SS communicates with a BS and how the UL packet scheduling works across different service classes. An initial request by the SS results in a bandwidth allocation by the BS to that SS. The SS packet scheduler is able dynamically to request a modification to its bandwidth allocation in a piggybacked requested to the BS. However, for simplicity of analysis in this paper it is assumed that the allocation remains static. As an SS may not have data available, it is polled by the BS. Polling may be on a group basis or in unicast fashion. As group polling may result in access delay at the SS, this paper assumes unicast polling, though there are UL sub-frame utilisation implications (Chang, 2008). The BS passes video and other data to the SS over the DL sub-frame whereas the UL sub-frame accommodates its traffic according to service class and burst slot availability. 


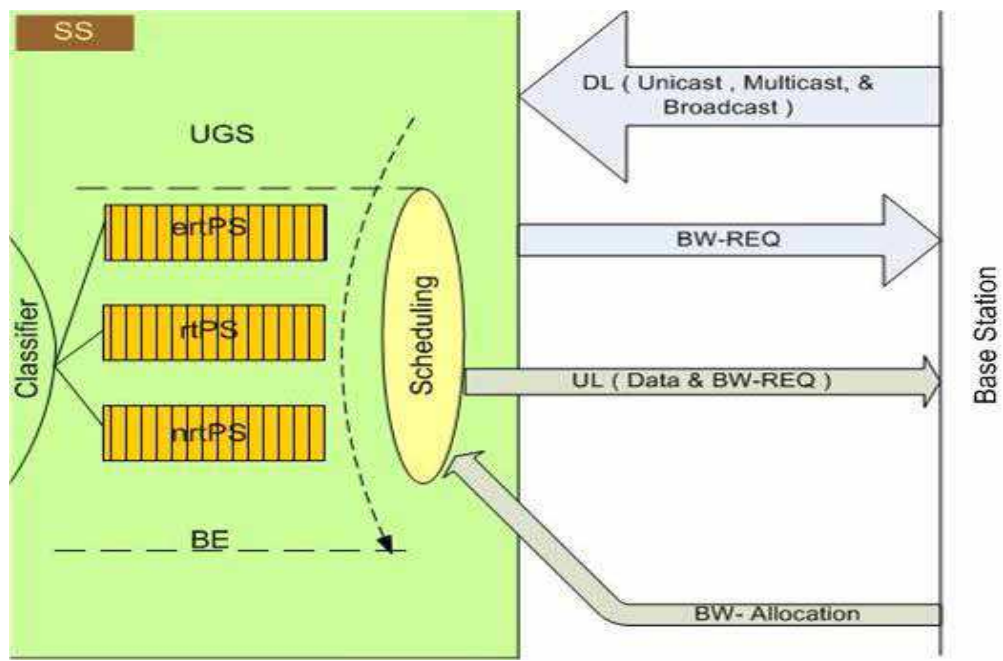

Fig. 12. IEEE 802.16 uplink scheduling system

The UGS is allocated bandwidth as of priority, while by default SS servicing of polled queues takes place in round-robin fashion (though many other possibilities exist [16]). In Figure 12 the queues are serviced by the packet scheduler in the order indicated with BE traffic considered last. In this Chapter, we assume an exhaustive service with the default simple round-robin access by the SS packet scheduler.

This Chapter concentrates on a particular class of SS UL queue discipline, Active Queue Management (AQM), as these have at least the potential to benefit real-time services by favoring traffic with urgent deadlines or with differing packet priorities (Orlov \& Necker, 2007). Within the fixed Internet, AQM techniques (Koo et al., 2004) are intended to achieve high link utilisation without introducing delays. The most well-known AQM technique is Random Early Detection (RED) (Floyd \& Jacobsen, 1993) in which packets in the queue are intentionally dropped or explicitly marked as a signal to the source to reduce its rate. For compliant sources, notably $\mathrm{TCP}$, a dropped packet prompts the congestion control mechanism to reduce its sending rate. For a non-compliant source, one that does not reduce its rate, RED (Floyd \& Jacobsen, 1993) also keeps the queue size low but does not discriminate against 'bursty' traffic sources. Dropping occurs in a probabilistic fashion according to the time-wise, exponentially-averaged queue size and no record is kept of the state of each connection to avoid unreasonable overhead. By random dropping, rate synchronization amongst compliant sources is avoided.

RED has a number of helpful features but it is known (Rahmani et al., 2008) to underperform when there are few co-existing flows in a queue because buffer occupancy fluctuates rapidly. In an SS, the number of coexisting flows could well be limited. RED maintains two thresholds: a low threshold in which all arriving packets are accepted and a high threshold when all arriving packets are rejected. When buffer fullness is between the thresholds, RED is in the congested state in which packets are randomly dropped. However, RED may not be able to react quickly enough to 'bursty' traffic operating within this region. Unfortunately, if buffer size is increased to compensate, apart from the drain on energy at 
any mobile device arising from the increased buffer size, interactive multimedia applications may suffer from a large buffer as a result of increased waiting times.

Random Exponential Marking (REM)'s packet marking probability (Athuraliya, 2001) grows considerably more aggressively than RED's. Its congestion measure is based on any mismatch between input rate and link capacity and between queue length and target length, though in practice rate change is measured by the rate of change of the queue length. The aim of REM is to stabilize the queue length to a target which is independent of traffic arrival intensity and of RED's queue thresholds. Like REM the BLUE algorithm (Feng, et al., 2002) also decouples queue length from congestion management. Rather than queue occupancy, BLUE employs a history of the current buffer packet overflow rate and link utilization to form the packet dropping (marking) probability. By updating its estimates over time, BLUE is able to learn the correct form of queue regulation. BLUE can also randomize times between marking probability updates to avoid source synchronization.

Drop-tail (FIFO) queue management (Clark et al., 1992), though not a form of AQM is ubiquitous across Internet routers and has the advantage that it scales well and shares delay between different connections. However, drop-tail may lock-out some connections to the advantage of others occupying the buffer. It also may maintain buffer fullness for longer periods of time, as it only signals congestion when the buffer is full.

\subsection{Video streaming from an SS}

In this Chapter, we consider two alternative ways of transporting video across an UL, the first through a basic UDP stream, representing a non-compliant source and the second through the TCP-Friendly Rate Control (TFRC) (Handley et al., 2003), representing a compliant source. TFRC is intended to provide a less aggressive congestion control than TCP's 'sawtooth' response to network congestion, resulting in a smoother sending rate more suitable for video streams. As a TFRC responds to packet loss, as well as round-trip time and packet size, it will change its rate if packets are actively dropped from an SS queue. TFRC has been widely studied in the fixed Internet and there is some interest in its use within wireless networks (Chen \& Zakhor, 2006). Because our interest is in queue management issues, the wireless channel is assumed to be error-free, while a detraction of TFRC for wireless networks is that it will also change its sending rate in response to packet losses on the channel, not just those resulting from congestion. However, in the event of channel loss, packet retransmission without acknowledgment to the TFRC application can avoid this problem, as it has for TCP in the SNOOP approach (Balkrishnan et al., 1997). Alternatively, in (Chen \& Zakhor, 2006) multiple TFRC connections are employed to dissipate channel packet loss on any one of the wireless connections.

In the simulations reported in Section 4.3, the reference 'Paris' clip was H.264 VBR-encoded at Common Intermediate Format (CIF) $(352 \times 288$ pixel/frame $)$ with 4:2:0 sampling and target bit-rate of $1.5 \mathrm{Mbps}$. The display rate was $30 \mathrm{~Hz}$. Paris consists of two figures seated around a table in TV studio format, with high spatial coding complexity. The Baseline profile was selected, as this is more easily supported by mobile devices because of reduced computational overhead. The Intra-refresh rate was every 15 frames with IPPP... structure. 1000 frames were transmitted. 


\subsection{Assessing uplink queuing disciplines}

WiMAX operating in point-to-multipoint mode was again simulated using the ns-2 simulator but this time through a WiMAX module from a Taiwanese research group (Tsai, 2006) that has proved an effective way of modeling WiMAX's behavior. The PHY settings selected for WiMAX simulation are given in Table 4, with the MAC settings defaulted from (Tsai et al., 2006). The DL/UL ratio is not intended to be realistic but to aid in testing the AQM algorithms, as in practice the DL would be allocated the majority of the bandwidth. The simulated configuration is shown in Figure 13. There were three SS communicating to the BS, with one of the SS sending VBR video encoded with the H.264/Advanced Video Codec (AVC). A trace file is input to ns-2 and packet losses recorded in the output. The output is employed to form the PSNR as a measure of video quality. Video quality comparisons were made under the EvalVid environment (Klaue et al., 2004).

\begin{tabular}{|l|l|}
\hline Parameter & Value \\
\hline PHY & OFDMA \\
Duplexing mode & TDD \\
Frame length & $5 \mathrm{~ms}$ \\
Bandwidth & $6 \mathrm{MHz}$ \\
FFT size & 1024 \\
DL/UL ratio & $1: 3$ \\
Fragmentation & yes \\
\hline
\end{tabular}

Table 4. Simulated WiMAX settings.

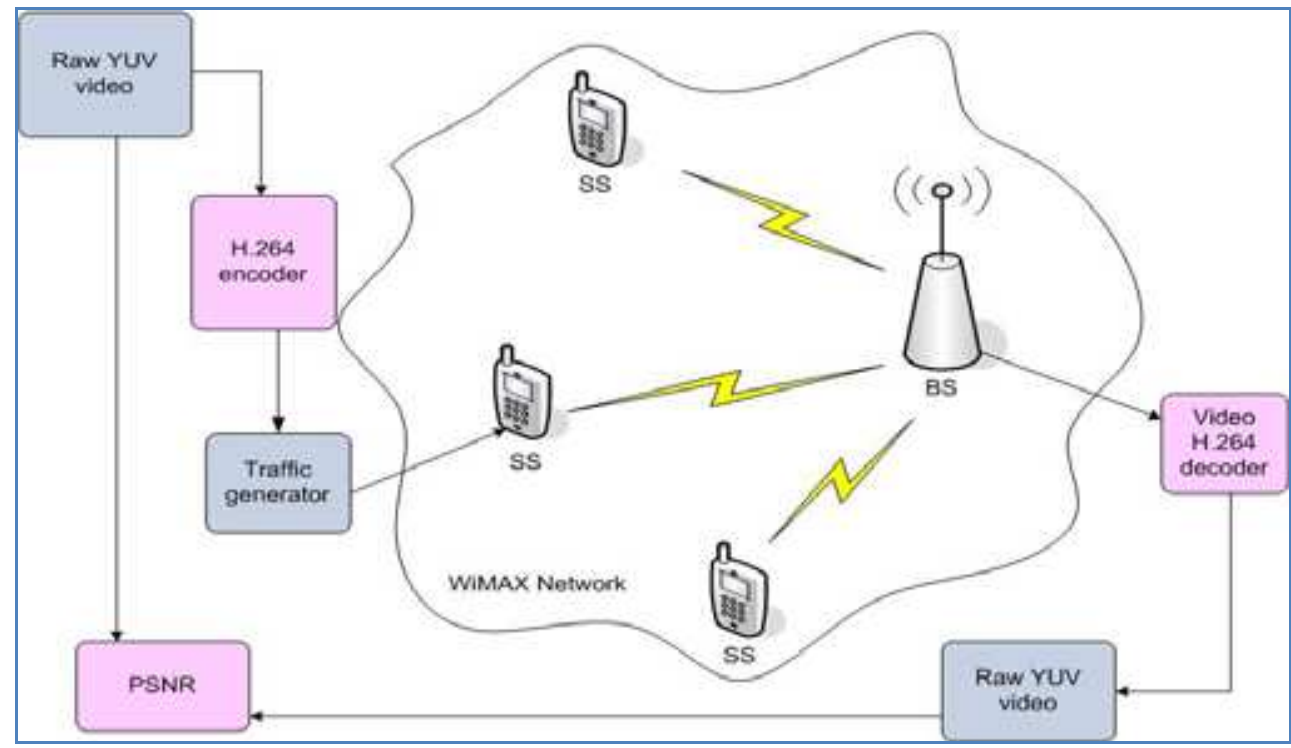

Fig. 13. IEEE 802.16 video performance simulation

Table 5 records the simulated traffic characteristics. As mentioned in Section 4.2, the video source was transported in two different ways: with simple UDP packetization and using 
TFRC. Network Adaptation Layer Units (NALUs) from H.264 were encapsulated with Real Time Protocol (RTP) headers on a single slice per frame basis. RTP includes a frame creation time-stamp allowing end-to-end delay to be estimated (see Section 5). However, the packet trace created by EvalVid (Klaue et al., 2003) from the H.264 output ensured packets did not exceed $1000 \mathrm{~B}$. This implies that there is some risk of decoder de-synchronization if a slice is split into several packets, causing slice de-synchronization markers to be missing from some packets. Under UDP transport, packets were transmitted with an inter-packet gap of $0.03 \mathrm{~s}$. For TFRC, the H.264 packet sizes were the same as for UDP but the inter-packet gap was varied according to the TFRC equation. As described in (Handley et al., 2003), TFRC is a receiver-based system in which the packet loss rate is found at the receiver and fed-back to the sender in acknowledgment messages. The sender calculates the round-trip delay from the acknowledgment messages and updates the packet sending rate. An equation that models TCP New Reno's sending rate is employed to find the sending rate. In a variant to standard TFRC, the packet size in the TFRC equation was dynamically altered according to the EvalVid-created trace file sizes. The underlying TFRC protocol is also UDP.

\begin{tabular}{|c|c|c|c|c|}
\hline SS-UL & Service type & $\begin{array}{c}\text { Traffic } \\
\text { type }\end{array}$ & Protocol & $\begin{array}{c}\text { Packet size } \\
\text { (B) }\end{array}$ \\
\hline \multirow{2}{*}{1} & rtPS & $\begin{array}{c}\text { VBR } \\
\text { (video) } \\
\text { CBR }\end{array}$ & UDP/ TFRC & Variable \\
& nrtPS & FTP & TCP & 1000 \\
\hline 2 & rtPS & CBR & UDP & 1000 \\
& nrtPS & FTP & TCP & \\
\hline 3 & rtPS & CBR & UDP & 1000 \\
& nrtPS & FTP & TCP & \\
\hline SS-DL & & & & \\
\hline $1,2,3$ & rtPS & CBR & UDP & 1000 \\
& nrtPS & FTP & TCP & \\
\hline
\end{tabular}

Table 5. Simulated traffic characteristics

Coexisting rtPS queue CBR sources were all sent at $1500 \mathrm{kbps}$, i.e. the same target rate as the video source. The inter-packet gap was also $0.03 \mathrm{~s}$ for the CBR traffic. The FTP applications were set up out of convenience as a way of occupying the nrtPS queues; otherwise a BE queue might be more appropriate. Likewise, the DL traffic is simply selected to fully occupy the DL link capacity.

The buffer size was set to fifty packets. This is not so large as to cause long queuing delays. Packets were dropped rather than explicitly marked at the queues, which will affect noncompliant applications, i.e. UDP, as well as compliant ones, i.e. TFRC. For RED, the dropping thresholds were set to $20 \%$ and $80 \%$ of the buffer size.

Tables 6 and 7 present mean video quality and end-to-end delay for UDP and TFRC transport. PSNR and delay were recorded on a per frame basis. From these Tables, it is apparent that TFRC definitely responds to packet drops at the rtPS queue and consequently improves the overall video quality. The mean delay for TFRC is also a little reduced compared to UDP transport, though the maximum delay is higher, due no doubt to TFRC managing its rate. REM's mean delay is less than the other queuing disciples suggesting a 
more aggressive regime which is confirmed by the lower video quality whether under UDP or TFRC transport, implying more packet drops. For TFRC, drop-tail queuing is actually preferable to the AQM queuing, while there is some advantage if UDP transport were to be used. From the Tables it is apparent that there is considerable variation in video quality on a frame-by-frame basis. The standard deviation (s.d.) is highest for TFRC under REM. The minimum per-frame PSNR values are unacceptable for viewing but the mean quality can be described as good, especially if received on a mobile station. However, our results do not take account of channel error, which would certainly reduce quality or delay (if some form of error control were applied).

\begin{tabular}{|c|c|c|c|c|c|}
\hline \multirow{2}{*}{$\begin{array}{c}\text { Queue } \\
\text { discipline }\end{array}$} & \multicolumn{3}{|c|}{ PSNR $(\mathrm{dB})$} & \multicolumn{2}{c|}{$\begin{array}{c}\text { End-to-end } \\
\text { delay (s) }\end{array}$} \\
\cline { 2 - 6 } & Mean & s.d. & Min. & Mean & Max. \\
\hline Drop Tail & 26.7 & 8.2 & 9.8 & 0.035 & 0.17 \\
RED & 29.8 & 9.1 & 10.7 & 0.035 & 0.17 \\
REM & 24.2 & 8.9 & 101 & 0.032 & 0.16 \\
BLUE & 29.8 & 9.1 & 10.7 & 0.036 & 0.16 \\
\hline
\end{tabular}

Table 6. Overall video quality and latency for UDP

\begin{tabular}{|c|c|c|c|c|c|}
\hline \multirow{2}{*}{$\begin{array}{c}\text { Queue } \\
\text { discipline }\end{array}$} & \multicolumn{3}{|c|}{ PSNR (dB) } & \multicolumn{2}{c|}{$\begin{array}{c}\text { End-to-end } \\
\text { delay (s) }\end{array}$} \\
\cline { 2 - 6 } & Mean & s.d. & Min. & Mean & Max. \\
\hline Drop Tail & 31.6 & 8.6 & 12.1 & 0.032 & 0.23 \\
RED & 31.4 & 8.8 & 12.2 & 0.033 & 0.24 \\
REM & 29.7 & 10.6 & 11.5 & 0.023 & 0.19 \\
BLUE & 31.4 & 8.8 & 12.2 & 0.033 & 0.24 \\
\hline
\end{tabular}

Table 7. Overall video quality and latency for TFRC

The packet drop figures were also compared, refer to Figure 14. As the total number of video packets sent was 4530, the dropped video packet numbers are around $10 \%$ of the whole. $10 \%$ packet loss is normally taken as recoverable from in video streaming (Agboma \& Liotta, 2008). The number of packets lost by the UDP stream was found to be greater than UDP for all queuing disciplines except REM. REM also balances its dropping rate between CBR sharing the rtPS queue and the video sources. However, REM's packet losses are relatively high. Other queuing disciplines have similar behaviors to each other, whether AQM or drop-tail.

Because encoded I-frames tend to be larger than encoded P-frames, after packetization to restrict the maximum packet size to $1000 \mathrm{~B}$, I-frames tend to form longer bursts of packets. From Figure 15, the percentage of I-frame packets dropped is larger than the percentage of P-frame packets (though the total of these is larger because there are more P-frames). Clearly this is a problem for REM and employing TFRC tends to result in a greater percentage of Iframe packet drops. This suggests that a drop policy based on preserving I-frame packets would benefit TFRC transport and could be applied using either RED or BLUE. 


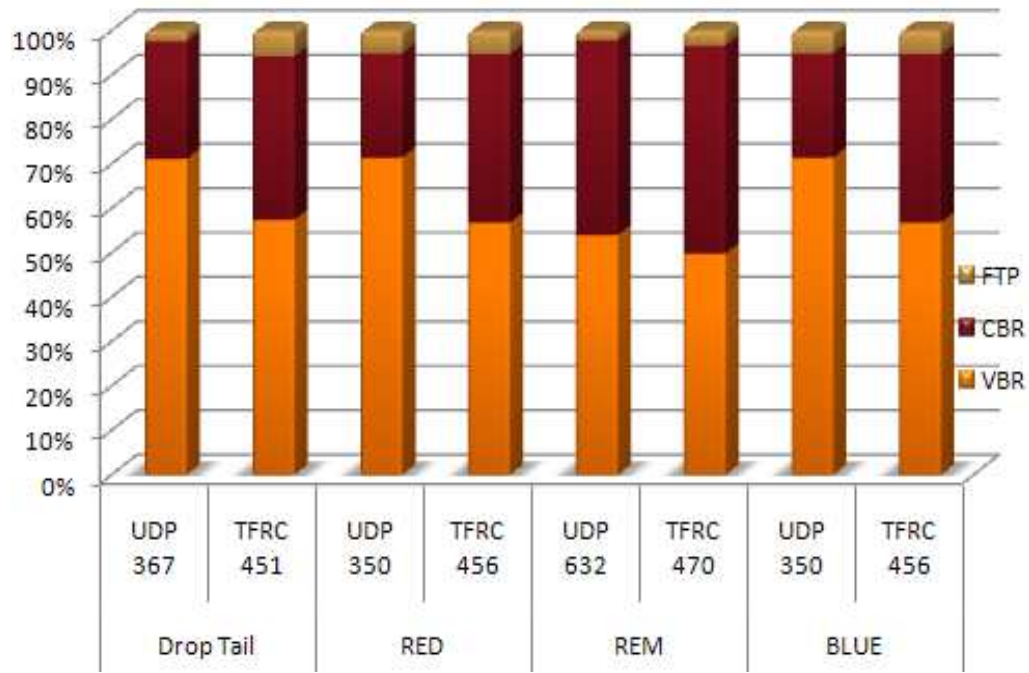

Fig. 14. Percentage packet drops according to traffic type and transport for differing queuing disciplines.

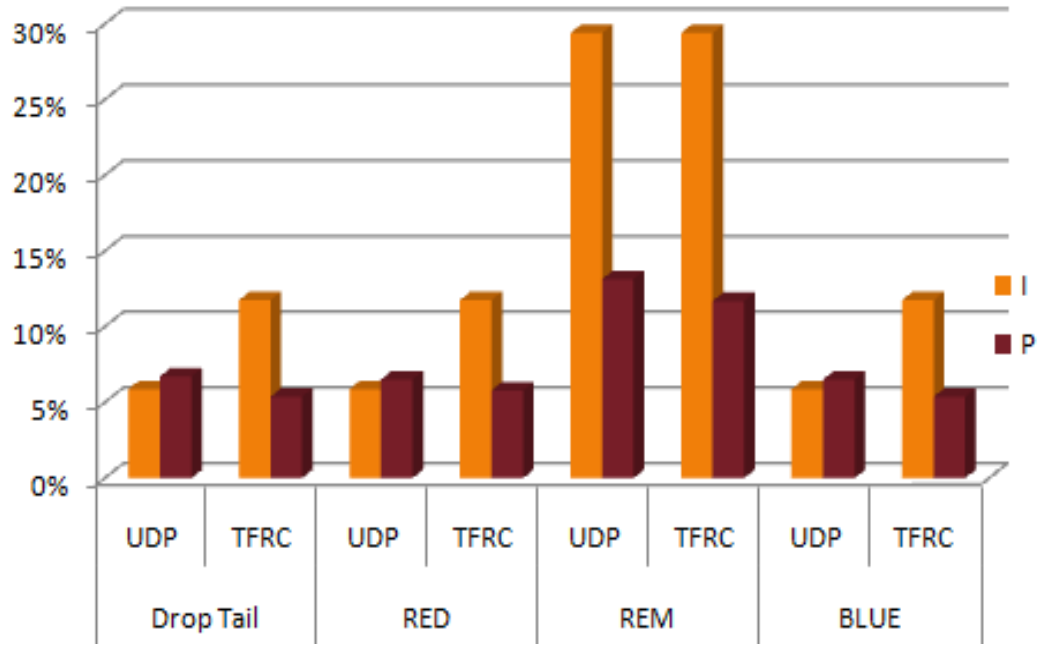

Fig. 15. Percentage of I- and P-frame packets dropped relative to numbers of I- and P-frame packets respectively

Apart from REM, drop-tail queuing and AQM for TFRC transport are equivalent in terms of the resulting mean video quality. Despite the detractions of using congestion controllers originally designed for the wired Internet, some form of congestion control is needed for WiMAX queues. REM is too aggressive, resulting in too many packet drops for 'bursty' traffic during periods of congestion. Because I-frame packets tend to arrive in bursts, there is a benefit from a dropping policy that favors I-frame packets. However, dropping predictive 
P-frame packets also has an enduring impact and given that B-frame packets are not available for this purpose in the low-complexity Baseline profile, some other content dependent queue management is required.

\section{H. 264 Packetization Issues}

H.264/Advanced Video Codec (AVC) (Richardson, 2003) is a compression tool that is able to offer better compression ratios for low-bit rate video than prior codecs. The reader is referred to (Wiegand et al, 2003), and other papers in the Special Issue on H.264 in which that paper appears for further information about H.264 itself, while in this Section we consider issues connected with packetisation.

Standardisation of H.264 was initiated by the Video Coding Experts Group (VCEG) which is a working group of the International Telecommunication Union (ITU-T). The Joint Video Team carried out the final work of developing the H.264, a co-operative effort between the Moving Picture Experts Group (MPEG) and VCEG in 2003. H.264 is divided into two parts: between a Video Coding Layer (VCL) and a Network Abstraction Layer (NAL). This allows VCL and NAL to be separately processed both when initially constructed and also when transported across a heterogeneous network. In the latter, the NAL could be reformed without any processing of the VCL content. For example, in wireline networks an Ethernet frame size of around 1500 B is typical of the Maximum Transport Unit (MTU) size, though this my vary across the public Internet but not within managed all-IP networks, whereas frame sizes nearer to $100 \mathrm{~B}$ are more typical of the MTU in wireless networks.

Briefly, the VCL represents the signal-processing functionality of the codec, with mechanisms motion compensation, frequency transform, loop filter and quantization. It is a macroblock-based coder that uses motion prediction between frames, with transform coding and motion compensation of the residual signal. Encoded macroblocks are collected into error resilience units called slices. A slice contains decoder resynchronisation markers and also indicates which control information is relevant to decoding the slice (parameter set). The output of the VCL consists of a bit-stream that containss the slice header (containing the spatial address of the first macroblock in the slice, the initial quantization parameter and similar information) and the macroblock data of an integer number of macroblocks. In H.264/AVC there are two types of slices: intra-frame coded and inter-frame coded. In the case of intra-frame coding, no previous or later frames are needed for encoding the present one, while in inter-frame coding previous and/or later frames are needed. Because H.264 allows prediction reference outside the boundary of a GOP, an Instantaneous Decoder Refresh (IDR) slice fulfills the role of an I-slice in previous codecs, in terms of a random access point for decoding, whereas an I-slice does not force the removal of prior references from the codecs' buffers.

VLC slices are encapsulated (Wenger et al., 2005) into Network Abstraction Layer Units (NALUs). Each NALU consists of a one-byte header and the payload bit string. The header refers to the type of the NAL unit, the (potential) appearance of bit errors or syntax violations in the NALU payload and other information related to the relative importance of the NALU for the decoding process. By identifying the importance of a NAL to error propagation avoidance, transmission software could judge the level of application-layer FEC to include. If transmission errors are possible (in a wireless network) then a 'forbidden' bit within the NAL to indicate to a decoder to apply error correction procedures (as most 
decoders contain some error detection and/or correction capability). An additional RealTime Protocol (RTP) (Schulzrinne et al., 1996) header is assumed, which is why the NALU is referred to as the RTP-payload.

The RTP header is $12 \mathrm{~B}$ long and conatins a sequence number, an application-specific timestamp such as the sampling instance, application-specific payload type, a marker bit for signalling the end of related packets such as those emitted at the same time. The interpretation of application-specific data may be negotiated through contraol data at the start of a streaming session. FEC data can additionally be sent in media-unaware RTP packets. Though consideration is beyond the scope of this Chapter, there will normally also be an outer transport-layer UDP packet of $8 \mathrm{~B}$, containing sequence number and a simple checksum. Congestion control for video streams is normally managed by an applicationlayer congestion controller, such as (refer to Section 4.2) TCP-Friendly Rate Control (TFRC) (Handley et al, 2003; Chen \& Zakhor, 2006). TFRC controls the rate at which UDP packets are emitted through a feedback mechanism form the receiver. Increasingly, and certainly for all-IP managed networks, a 20 B IP header is also included to bring the total non-video header overhead to $40 \mathrm{~B}$.

In the default case, one NALU contains a single coded slice. However, for very large slices such as might occur in high definition digital cinema, there is an option to sequence NAL fragments eah within its own RTP packet. Conversely, some NALUs are only a few bytes in size and may be aggregated within a single RTP packet, either all with the same timestamp or with differing timestamps, which must be coded relative to the RTP header's timestamp.

Addtionally it is possible to partition a slice. When using partitioning of data, a single slice of coded data can be divided in up to three parts, with each part put in a separate NALU. The encoder can be set so that each partiction does not exceed the MTU. The definition of the partitioning for a $\mathrm{P}$ - slice is as follows:

1- A partition has a slice header, quantization parameter, macroblock type, mode of prediction and motion vectors.

2- B partition contains the intra-coded macroblocks residual information.

3- C partition has the inter-frame coded macroblocks residual information.

Notice that some macroblocks in a pre-dominantly inter-coded slice may be predicted in an intra-coded (spatially predicted) manner in order to correct error propagation but as these are usually few it may be preferable to merge B- and C-partitions to avoid small NALUs. However, if a encoder is designed for wireless channels then more intra-coded macroblocks may be inserted and in which case separate B and C NALUs may be more appropriate (Stockhammer \& Bystrom, 2004). The 3 partition arrangement differs from MPEG-4 and H263, which only support two partitions. As I-slices in H.264 do not contain inter-frame coded blocks, C partitions do not occur, while B-slices have only partition.

The ability to use information from a correctly received partition when one of the other partitions is lost is the main idea of data partitioning. To be capable of doing this, it is important to be aware of the differing dependencies between the partitions. Each of partitions B and C cannot be decoded when partition A is lost, because without the motion vectors and prediction mode, the decoder cannot decode macroblocks. On the other hand, partition A information, especially the motion vectors, could be helpful to the decoder to compensate for the loss of either or both of the corresponding B and C partitions. We can conclude that partition $\mathrm{A}$ is independent of both partitions $\mathrm{B}$ and $\mathrm{C}$, while the reverse is not correct. Therefore, the best achievable quality of decoded video arises if the proabability of 
losing partition A is smaller than the probability of losing both partitions B and C. This is can be effected (Wenger, 2003; Stockhammer \& Bystrom, 2004) by either using UEP or by sending partition A by a side channel.

In (Ferre et al., 2008), a packetization method was presented for robust H.264 video transmission over IEEE 802.11 wireless local area network (WLAN) configured as a home network. This research, though not applied to WiMAX does illustrate how careful handling of H.264's NALUs can improve video quality. Video robustness was enhanced by using small NALUs each of which was encapsulated (after addition of RTP header) into an IP packet. Then several NALU-bearing IP packets were mapped into a larger MAC frame. By retrieving error-free IP packets from within the received MAC frame greater robustness to error was achieved. The NAL aggregation mechanism with packet recovery was evaluated via simulation. The system provided a $2.5-\mathrm{dB}$ gain in video quality for similar throughput and an $80 \%$ improvement in throughput efficiency was achieved for a similar video quality (PSNR) video performance. However, this method seems less efficient than the NAL aggregation method which requires a single IP header.

The research reported in (Chatterjee, 2007) used FEC and ARQ techniques to support streaming over WiMAX without violating the standard. The channel state information was employed to dynamically construct the MPDU. The sizes of these units were determined such that the packet dropping probability was minimized without compromising the goodput. Simulation results showed the performance enhancements achieved by the proposed ARQ-enabled adaptive algorithm for streaming data.

A Digital Cinema video distribution architecture with the motion JPEG codec is discussed in (Micanti et al., 2008). Though the issue of the need to avoid retransmission in the packetization strategy is discussed, this is on a general level and tests appear to only be for 802.11 rather than 802.16 as the title suggests. The scheme suggests careful mapping of the codestream structure onto the underlying transport packets.

Finally in (Stockhammer \& Baystrom, 2004), the researchers applied data partitioning under H.264/AVC for conversational applications (e.g. videophone, videoconferencing) over mobile wireless channels. The experimental results showed that the probability of entirely lost frames was lowered and the probability of poor quality decoded video was reduced by the data-partitioning scheme. In this scheme, if FEC fails to protect an A partition then previous frame replacement is applied, while if a B- but not an A-partition is lost temporal error concealment is employed.

\section{Conclusion}

This Chapter has presented techniques to achieve successful video streaming over WiMAX networks. Because WiMAX queue management and scheduling are in the gift of the developer, it is possible to devise schemes that are either multimedia-friendly or do not discriminate against the requirements for video streaming. Because WiMAX supports OFDM, UEP through adaptive modulation is possible and, because there are many flexibilities in the WiMAX frame and MPDU, it is possible to may the video stream output onto these structures. The Chapter described research in adaptive modulation based on prioritising between frame types and uplink SS queue management. The necessary background for manipulation of H.264 NALUs, especially through data-partitioning was presented, though investigations are at an early stage. It is possible that all these techniques 
can be combined and there are other possibilities supported by H.264 such as smooth switching between streams of different quality according to channel conditions. As in all applications of video streaming over wireless, WiMAX technology represents a rich field of investigation.

\section{Acknowledgment}

We gratefully thank B. Tanoh for conducting simulations for R. Razavi to verify the results in Section 3 of this Chapter.

\section{References}

Agnoma, F.; \& Liotta, A. (2008) QoE Analysis of a Peer-to-Peer Television System. In Proceedings of IADIS Int. Conf. on Telecomms, Networks and Systems

Ahson, A. \& Ilyas, M. (eds.) (2008) WiMAX Applications, Taylor \& Francis Group, ISBN 9781-4200-4547-5, Boca Raton, FL

Ali, N.A., Dhrona, P. \& Hassanein, H. (2009) A Performance Study of Uplink Scheduling Algorithms in Point-to-Multipoint WiMAX Networks, Computer Comms., Vol. 32, No. 3, pp. 511-521

Anderson, H. R. (2003) Fixed Broadband Wireless System Design, Wiley \& Sons, ISBN 0-47084438-8, Chichester, UK

Andrews, J. G.; Ghosh, A. \& Muhamed, R. (2007) Fundamentals of WiMAX: Understanding Broadband Wireless Networking, Prentice Hall, ISBN 0-13-222552-2, Upper Saddle River, NJ

Athuraliya, S.; Li, V.H.; Low, S.H. \& Yin, Q. (2001) REM: Active Queue Management. IEEE Network, Vol. 15, No. 2, (48-53)

Balkrishnan, H.; Padmanabhan, V.; Seshan, S. \& Katz, R. (1997) A Comparison of Mechanisms for Improving TCP Performance over Wireless Links, IEEE/ACM Trans. On Networking, Vol. 5, No. 6, pp. 756-769

Chang, B.-J.; Chou C.-M.; \& Liang, Y.-H. (2008) Markov Chain Analysis of Uplink Subframe in Polling-based WiMAX Networks, Computer Comms., Vol. 31, No. 10, pp. 23812390

Chatterjee, M.; Sengupta, S. \& Ganauly, S. (2007) Feedback-Based Real-Time Streaming over WiMAX, IEEE Wireless Commun., Vol. 14, No. 1, pp. 64-71

Chen, C.-M.; Lin, C. -W. \& Chen, Y.-C. (2006) Unequal Error Protection for Video Streaming Over Wireless LANs using Content-Aware Packet Retry Limit, In Proceedings of IEEE Int. Conf. on Multimedia and Expo, pp. 1961-1964

Chen, M. \& Zakhor, A. (2006) Multiple TFRC Connections Based Rate Control for Wireless Networks. IEEE Trans. On Multimedia, Vol. 8, No. 5, pp. 1045-1061

Clark, D.D.; Shenker, S. \& Zhang, L. (1992) Supporting Real-time Applications in an Integrated Services Packet Network: Architecture and Mechanism, SIGCOMM '92, pp. $14-26$

Ercge, V.; Hari, K. V. S. et al., (2001) Channel Models for Fixed Wireless Applications, Contribution IEEE 802.16.3c-01/29rl Feb. 2001

Feng, W.-C.; Shin, K. G.; Dilip, D. K; \& Saha, D. (2002) Active Queue Management Algorithms, IEEE/ACM Trans. on Networking, Vol. 10, No. 4, pp. 513-528 
Ferre, P.; Doufexi, A.; Chung-How, J.; Nix, A. R. \& Bull, D. R. (2008) Robust Video Transmission over Wireless LANs, IEEE Trans. on Vehicular Technol., Vol. 57, No. 4, pp. 2596- 2602

Floyd, S. \& Jacobson, V. (1993) Random Early Detection Gateways for Congestion Avoidance, IEEE/ACM Trans. on Networking, Vol. 1, No. 4, pp. 397-413

Ghanbari, M. (2003), Standard Codecs: Image Compression to Advanced Video Coding, IET Press, Stevenage, ISBN 0-85296-710-1, UK

Handley, M.; Pahdye, J.; Floyd, S. \& Widmer, J. (2003) TCP-Friendly Rate Control (TFRC): Protocol Specification. RFC 3448.

Hanzo, L. \& Choi, B.-J. (2007) Near-Instantaneously Adaptive HSDPA-Style OFDM Versus MC-CDMA Transceivers of WiFI, WiMAX, and Next-Generation Cellular Systems, Proceedings of the IEEE, Vol. 95, No. 12, pp. 2368-2392

Hillestad, O. J.; Perkis, A.; Genc, V.; Murphy, S. \& Murphy, J. (2006) Delivery of OnDemand Video Services in Rural Areas via IEEE 802.16 Broadband Wireless Access Networks, In Proceedings of 2nd ACM Workshop on Wireless Multimedia Networking and Performance Modeling, pp. 43-51

Honig, M. K. \& Messerschmitt, D. G. (1990) Adaptive Filters Structures, Algorithms, and Applications, Kluwer, ISBN 978-0-898-38163-4, Boston, MA

Hoymann, C. (2005) Analysis and Performance Evaluation of the OFDM-based Metropolitan Area Network IEEE 802.16, Computer Networks, Vol. 49, pp. 341-363

Juan, H. H.; Huang, H.-C.; Huang, C.-Y. \& Chiang, T. (2007) Scalable Video Streaming over Mobile WiMAX, IEEE Int. Symposium on Circuits and Systems, pp. 3463-3466.

Klaue, J.; Rathke, B. \& Wolisz, A. (2003) EvalVid - A Framework for Video Transmission and Quality Evaluation, In Proceedings of Int. Conf. on Modeling Techniques and Tools for Computer Performance, pp. 255-272

Koo, J.; Ahn, S.; \& Chung, J. (2004) Performance Analysis of Active Queue Management Schemes for IP Network, In Proceedings of Int. Conf. on Computational Science, pp. 349-356

Li, Q. \& Schaar, M. van der (2004) Providing QoS to Layered Video Over Wireless Local Area Networks Through Real-Time Retry Limit Adaptation, IEEE Trans. on Multimedia, Vol. 6, No. 2, pp. 278-290

Meloni, L. G. P. (2008) A New WiMAX profile for DTV Return Channel and Wireless Access., pp. 291-392, In Mobile WiMAX, Chen, K.-C. and de Marca, J. R. B. (eds.), Wiley \& Sons, ISBN 978-0-470-51941-7, Chichester, UK

Micanti, P.; Baruffa, G. \& Fabrizio Frescura, F. (2008) A Packetization Technique for DCinema Contents Multicasting over Metropolitan Wireless Networks, pp. 313-328, In Mobile WiMAX, Chen, K.-C. and de Marca, J. R. B. (eds.), Wiley \& Sons, ISBN 978-0-470-51941-7, Chichester, UK

Milanovic, J.; Rimac-Drlje, S. \& Bejuk, K. (2007) Comparison of Propagation Models Accuracy for WiMAX on $3.5 \mathrm{GHz}$, In Proceedings of IEEE Int. Conf. on Electronics, Circuits and Systems, pp. 1111-1114

Negi, R.; \& Cioffi, J. (1998) Pilot Tone Selection for Channel Estimation in a Mobile OFDM System, IEEE Trans. On Consumer Electron., Vol. 44, No. 3, pp. 1122-1128

Nuaymi, L. (2007) WiMAX: Technology fo Broadband Wireless Access, Wiley \& Sons, ISBN 9780-470-02808-7, Chichester, UK 
Orlov, Z. \& Necker, M. C. (2007) Enhancement of video streaming QoS with Active Buffer Management in Wireless Environments, In Proceedings of $1^{\text {th }}$ European Wireless Conf.

Rahmani, R., Hjelm, M. and Aklund, C. (2008) Active Queue Management for TCP Friendly Rate Control Traffic in Heterogeneous Networks, In Proceedings of Int. Conf. on Telecoms., pp. 1-7

Richardson, I. E. (2003) H.264 and MPEG-4 Video Compression, Wiley \& Sons, ISBN 0-47084837-5, Chichester, UK

Razavi, R., Fleury, M. \& Ghanbari, M. (2008) Unequal Protection of Video Streaming through Adaptive Modulation with a Tri-Zone Buffer over Bluetooth EDR, EURASIP J. on Wireless Comms. And Networking, 16 pages, online vol.

Sadka, A. (2002) Compressed Video Communications, Wiley \& Sons, ISBN 0-470-84312-8, Chichester, UK

Schaar, M. van der; Chou, P. A. (eds.) (2007) Multimedia over IP and Wireless Networks, Academic Press, ISBN 13-978-0-12-088480-3, Burlington, MA

Schulzrinne, H.; Casner, S.; Frederick, R. \& Jacobson, V. (1996) RTP: A Transport Protocol for Real-Time Applications, RFC 1889, 1996

Stockhammer, T. \& Baystrom, M. (2004) H.264/AVC Data Partitioning for Mobile Video Communication, In Proceedings of Int. Conf. on Image Processing, pp. 545-548

Tariq, U.; Jilani, U. N. \& Siddiqui, T.A. (2007) Analysis on Fixed and Mobile WiMAX, MSc Thesis Report, Blekinge Institute of Technology, Sweden

Tsai, F. C.-D., et al., (2006) The Design and Implementation of WiMAX Module for ns-2 Simulator. Workshop on ns2, article no. 5

Wenger, S. (2003) H.264/AVC Over IP, IEEE Trans. on Circuits and Syst. for Video Technol., Vol. 13, No. 7, pp. 645-656

Wenger, S.; Hannuksela, H. H.; Stockhammer, T.; Westerlund, M. \& Singer, R. (2005) RTP Payload Format for H.264 Video, RFC 3984

Wiegand, T.; Sullivan, G. J.; Bjontegaard, G. \& Luthra, A. (2003) Overview of the H.264/AVC Video Coding Standard, IEEE Trans. on Circuits and Syst. for Video Technol., Vol. 13, No. 7, pp. 560-576

Yun, J. \& Kavehard, M. (2006) PHY/MAC Cross- Layer Issues in Mobile WiMAX, Bechtel Telecomm. Techn. Journal, Vol. 4, No. 1, pp. 45-56

Zhang, H.; Li, Y.; Feng, S. \& Wu, W. (2006) A New Extended rtPS Scheduling Mechanism Based on Multi-polling for VoIP Service in IEEE 802.16e System, In Proceedings of Int. Conf. on Communication Technol., pp. 1-4 


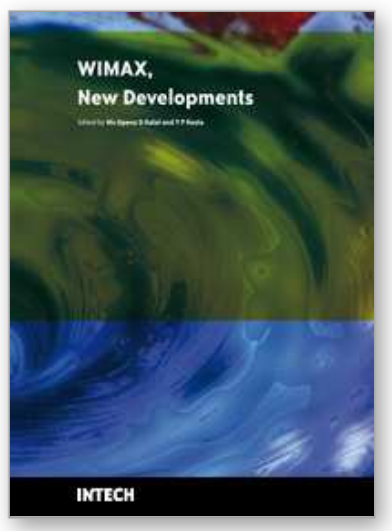

\author{
WIMAX New Developments \\ Edited by Upena D Dalal and Y P Kosta
}

ISBN 978-953-7619-53-4

Hard cover, 442 pages

Publisher InTech

Published online 01, December, 2009

Published in print edition December, 2009

WiMAX (Worldwide Interoperability for Microwave Access) is a wireless broadband access network named by industry group called the WiMAX forum formed in June 2001. It is Wireless MAN with IEEE 802.16 family standards. Loosely, WiMAX is a standardized wireless version of Ethernet that enables the last mile, intended primarily as an alternative to wire technologies (such as Cable Modems, DSL and T1/E1 links ) to provide broadband access to customer premises. Mission of the WiMAX forum is to promote and certify compatibility and interoperability of broadband wireless products. This book touches most of the above issues in form of 22 individuals' papers containing research work in WiMAX domain in particular. WiMAX has two important standards/usage models: a fixed usage model IEEE 802.16-2004 for Fixed Wireless Broadband Access (FWBA) and a portable usage model IEEE 802.16e-2005, which is mainly concentrated on Mobile Wireless Broadband Access (MWBA). Both are released as standards and amendments are available in form of drafts. Higher data rate transmissions (@ 100 Mbps) are achieved in IEEE 802.16-2004 WiMAX through LOS communications which incorporate a stationary transmitter and receiver but IEEE 802.16e supporting NLOS communication is much complicated and little less bit rate is achieved. $2-11 \mathrm{GHz}$ licensed band is the range of frequencies with TDD and FDD supports. The book will provide a wide horizon to visualize the WiMAX technology and its developments leading towards $4 \mathrm{G}$ systems. It will provide a good platform to the researchers with clues to the innovative ideas in WiMAX domain. I wish all the best to the authors and readers of this book in their successful research of WiMAX technology.

\title{
How to reference
}

In order to correctly reference this scholarly work, feel free to copy and paste the following:

M. Fleury, R. Razavi, S. Saleh, L. Al-Jobouri and M. Ghanbari (2009). Enabling WiMAX Video Streaming, WIMAX New Developments, Upena D Dalal and Y P Kosta (Ed.), ISBN: 978-953-7619-53-4, InTech, Available from: http://www.intechopen.com/books/wimax-new-developments/enabling-wimax-video-streaming

\section{INTECH}

open science | open minds

\section{InTech Europe}

University Campus STeP Ri

Slavka Krautzeka 83/A

51000 Rijeka, Croatia

Phone: +385 (51) 770447

Fax: +385 (51) 686166

\section{InTech China}

Unit 405, Office Block, Hotel Equatorial Shanghai

No.65, Yan An Road (West), Shanghai, 200040, China 中国上海市延安西路65号上海国际贵都大饭店办公楼 405 单元

Phone: +86-21-62489820

Fax: +86-21-62489821 
www.intechopen.com 
(C) 2009 The Author(s). Licensee IntechOpen. This chapter is distributed under the terms of the Creative Commons Attribution-NonCommercial-ShareAlike-3.0 License, which permits use, distribution and reproduction for non-commercial purposes, provided the original is properly cited and derivative works building on this content are distributed under the same license. 